\title{
Monotonous and Massive: Small Lymphocytic Lymphoma
}

\section{Anubha Bajaj*}

AB Diagnostics, Hospital and Health Care, West Delhi, Delhi, India

\begin{abstract}
Chronic Lymphocytic Leukaemia (CLL) may be designated as a frequent adult B cell leukemia of the developed world, detected at a median age of 72 years. An Absolute Lymphocyte Count (ALC) of mature-appearing lymphocytes greater than 5000 cells/ $\mu \mathrm{L}$ with a B lymphocyte immune reactivity to CD20+,CD5+, CD19+, CD23+,CD43+, CyclinD1, monoclonal immunoglobulins such as immunoglobulin $\mathrm{M}$ and $\mathrm{D}$ (IgM and $\lg \mathrm{D}$ ), and a kappa/lambda light chain restriction of the surface immunoglobulin may be delineated. Small Lymphocytic Lymphoma (SLL) may be categorized where the ALC remains below 5000 cells $/ \mu \mathrm{L}$. The genetic discrepancies detected on Fluorescent in Situ Hybridization (FISH) enunciate a 17p deletion, 11q deletion, 12q trisomy, a normal genotype or a solitary chromosomal 13q deletion. The histology of SLL may exhibit an obliterated nodal architecture with a predominant, monotonous infiltrate of innumerable, miniature, round lymphocytes exhibiting aggregated chromatin, indistinct nucleoli and scanty cytoplasm with minimal mitosis. A blastic, histiocytic or a malignant conversion with enlarged cells may manifest with SLL or CLL, the phenomenon may be designated as "Richter's syndrome" and signifies an accelerated clinical course with a declining survival.Z
\end{abstract}

\begin{abstract}
Abbreviations: ALC: Absolute Lymphocyte Count; SLL: Small Lymphocytic Lymphoma; FISH: Fluorescent in Situ Hybridization; CLL: Chronic Lymphocytic Leukaemia; CT: Computerized Tomography; IGHV: Immunoglobulin Heavy Chain Variable; ORR: Objective Response Rate; PFS: Progression Free Survival
\end{abstract}

\section{Preface}

A frequent form of adult leukemia may be manifest by an accretion of functionally incompetent lymphocytes gradually within the peripheral blood, bone marrow, spleen and lymph nodes which may be designated as a CLL. An annual estimation of 1500 instances may be elucidated with CLL in the developed world. The disease may be detected coincidentally at a median age of 72 years or may clinically manifest with a coexistent tumor encumbrance, autoimmune disorders or infections. An ALC of mature-appearing lymphocytes greater than $5000 \mathrm{cells} / \mu \mathrm{L}$ with an immune reactivity to $\mathrm{CD} 5+, \mathrm{CD} 19+, \mathrm{CD} 23+$, and a kappa/lambda light chain restriction of the surface immunoglobulin may be delineated. The disorder may be labeled as SLL in situations where the ALC persists below 5000 cells $/ \mu \mathrm{L}$ [1]. SLL frequently arises in the middle-aged or elderly individuals and generally elucidates a favorable outcome. It may be discovered coincidentally in lymph nodes examined for suspected carcinomas. The condition may usually be discovered at a median age of 72 years. An estimated $10 \%$ of the implicated individuals may be first degree relatives of CLL patients or below 50 years of age [1]. The disease demonstrates a male predominance with a male to female ratio (M:F) of 2:1. The environmental factors engendering the lymphocytic transformation may be indeterminate, though the exposure to agent orange in specific war veterans may be considered [1].

\section{Constitutional Analogy}

The disorder may appear to be asymptomatic. Indeterminate symptoms such as night sweats, fever, fatigue, weight loss, lymph node or spleen enlargement may be encountered. Bone marrow may be implicated with the emergence of hematological cytopenias, nonspecific, generalized infection or a dermal pathology. An elevation of the lymphocyte counts may not elucidate specific symptoms $[1,2]$.

Three categories of SLL may be elucidated with distinctive clinical attributes:
1.) An increase in the lymphocyte count with absolute lymphocytosis (designated as CLL)

2.) A definitive monoclonal gammopathy with an estimated $50 \%$ instances enunciating lesions of the bone marrow

3.) A clinical scenario concordant with an absence of absolute lymphocytosis or monoclonal gammopathy, although hypogammaglobulinemia may coexist [2]. The three divergent sub-types may not depict a significant variation of the survival percentages. The first and the third subtypes may depict identical morphology

\section{Molecular Modifications}

The genetic discrepancies detected on FISH enunciate a 17p del, $11 \mathrm{q}$ del, 12q trisomy, a normal genotype or $13 \mathrm{q}$ del. A majority (80\%) of the CLL patients may depict 17p-, 13q- and a $+12 \mathrm{q} 13$ genomic aberrations. Additional alterations of immunoglobulin heavy chain variable region (Ig VH), CD38, Zap 70 with increased serum $\beta 2$ microglobulin levels and soluble CD23 may be delineated. Variable B cell regions with rearrangement and mutation within the $\mathrm{VH}, \mathrm{D}, \mathrm{JH}$, and $\mathrm{C} \mu$ may be elucidated $[1,2]$.

\section{Diagnostic Determinants}

- A complete peripheral blood count with differential leucocyte count may be acquired

- Serum chemistries such as the liver function tests, renal function tests, serum Lactate Dehydrogenase $(\mathrm{LDH})$ with serum $\beta 2$

*Corresponding author: Anubha Bajaj, AB Diagnostics, Hospital and Health Care, West Delhi, Delhi, India, E-mail: anubha.bajaj@gmail.com

Received September 27, 2018; Accepted October 20, 2018; Published October 28, 2018

Citation: Bajaj M (2018) Monotonous and Massive: Small Lymphocytic Lymphoma. J Integr Oncol 7: 217. doi: 10.4172/2329-6771.1000217

Copyright: (C) 2018 Bajaj M. This is an open-access article distributed under the terms of the Creative Commons Attribution License, which permits unrestricted use, distribution, and reproduction in any medium, provided the original author and source are credited. 
microglobulin levels with a quantification of serum immuneglobulins may be mandated

- A quantifiable lymphocyte percentage of greater than $30 \%$ on a bone marrow aspiration may be diagnostic, thereby excluding the necessity of a bone marrow biopsy. Bone marrow biopsy and aspiration may not be beneficial if the hematological parameters are within the normal range [3-8]

- Flow cytometry and FISH analysis of peripheral blood may confirm the preliminary diagnosis of CLL. The procedure may also provide prognostic information along with decisive therapeutic options $[2,3]$

- A Computerized Tomography (CT) scan may be mandated with the appearance of constitutional symptoms. A Positron Emission Tomography (PET) scan may be desirable when the "Richter's Syndrome" is suspected

- Interphase cytogenetics may also be employed for ascertaining anomalies such as trisomy +12 , del 13q, del 17(p13.1) and del 11 $\mathrm{q} 22.3$. Alterations such as del $17 \mathrm{p}$ and del $11 \mathrm{q}$ may exemplify an aggressive condition. An un-mutated Immunoglobulin Variable Heavy genetic assay along with $\beta 2$ microglobulin levels may be accomplished. Analysis of a Zap 70 by flow cytometry may be beneficial and recommended only with clinical trials [3,4,7]. Somatic hyper-mutations may be detected on genetic analysis

- A cytogenetic analysis with FISH may delineate chromosomal aberrations such as 17 pdeletion, 11q deletion, a 12q trisomy, a normal karyotype or a singular anomaly of chromosomal $13 \mathrm{q}$ deletion

\section{Histological Elucidation}

The nodal architecture may be obliterated with a predominant, monotonous infiltrate of innumerable, miniature, round lymphocytes exhibiting aggregated chromatin, indistinct nucleoli and scanty cytoplasm with minimal mitosis [2]. Solitary or accumulated, diverse and enlarged cells such as the pro-lymphocytes and para immunoblasts with vesicular nuclei and conspicuous nucleoli may simulate germinal centers [5,9]. The particular configuration may be termed as a proliferation center, growth center or a pseudo-follicle and may exhibit elevated Ki 67 reactivity. The articulation a distinction from a follicular lymphoma or a nodular lymphocytic predominance Hodgkin's Lymphoma [2]. The emergence of pseudo-follicles and/or pro lymphocytes with para immunoblasts may corroborate the occurrence of an SLL, in contrast to a mantle cell lymphoma. A particular variant with preponderantly diffuse disease and circumscribed, benign lymphoid follicles may be denominated as the "inter-follicular SLL" and may be restricted to the marginal zone, the peri-follicular or interfollicular areas [2]. An estimated one-third of the instances of SLL may exemplify an extra-nodal invasion [5,10] (Figures 1-14).

In concordance with monoclonal gammopathy, the malignant lymphocytes may be admixed with mature plasma cells with the morphologic attributes of a plasmacytoid differentiation such as an oval cellular outline, a laterally displaced nucleus, a distinct peri-nuclear hof and pyroninophilia. The nodal architecture may be partially preserved. The modification may be designated as "SLL with plasmacytic differentiation" [2].

\section{Immune Histochemistry}

It may exhibit the small lymphocytic lymphoma to consistently

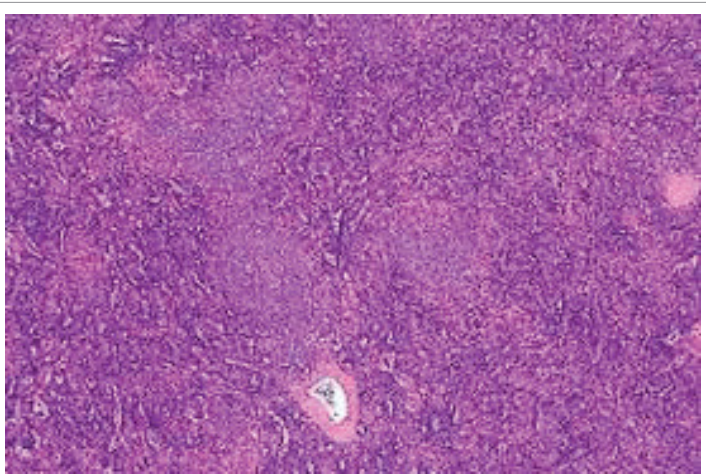

Figure 1: SLL-effaced nodal architecture with monotonous lymphoid infiltrate [20].

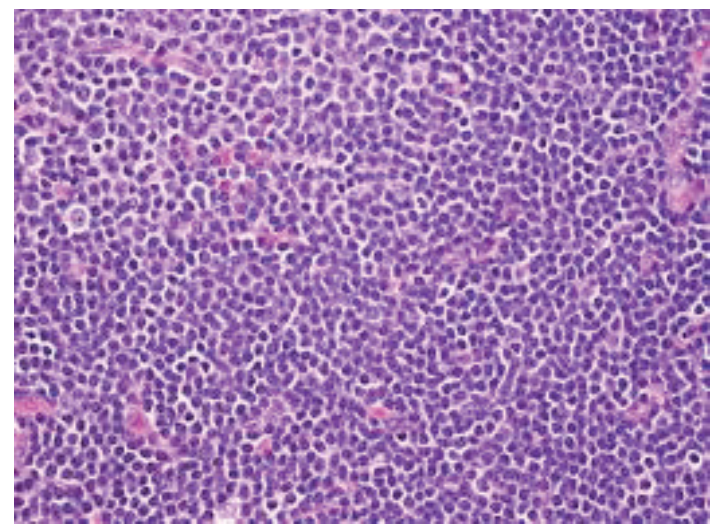

Figure 2: SLL-homogenous impregnation of I lymphoid population [21].

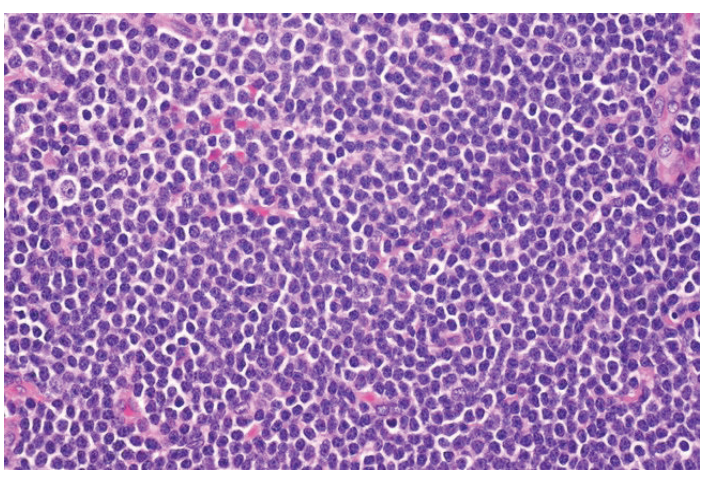

Figure 3: SLL-tiny, mature lymphoid cells with a uniform distribution [22]

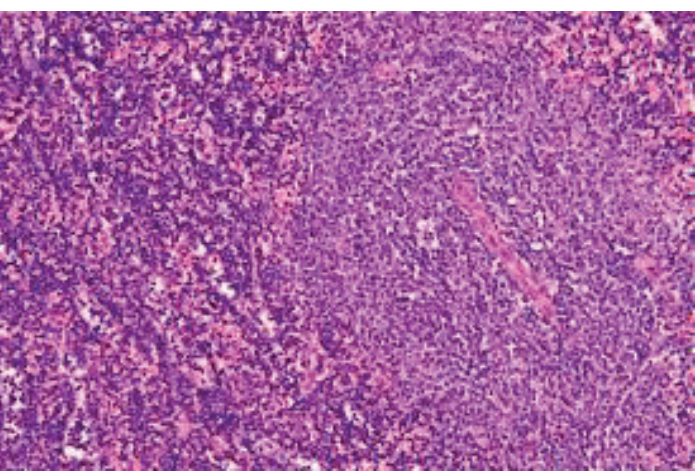

Figure 4: SLL-intense accrual of small lymphoid cells [23]. 


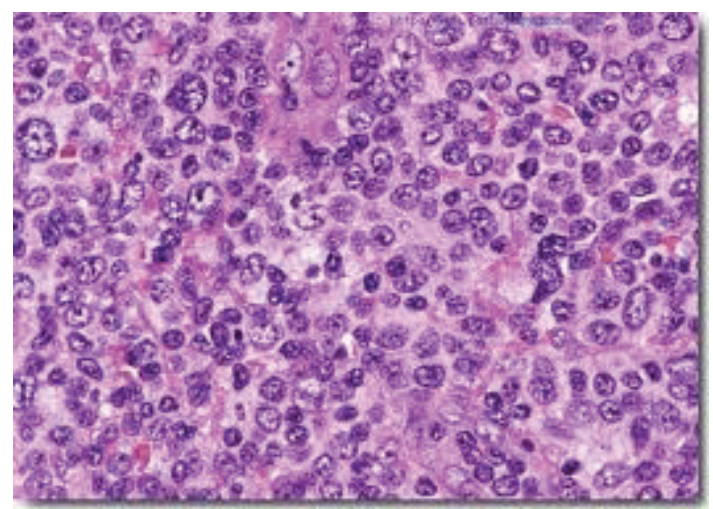

Figure 5: SLL-activated lymphocytes with vesicular nuclei and prominent nucleoli [24].

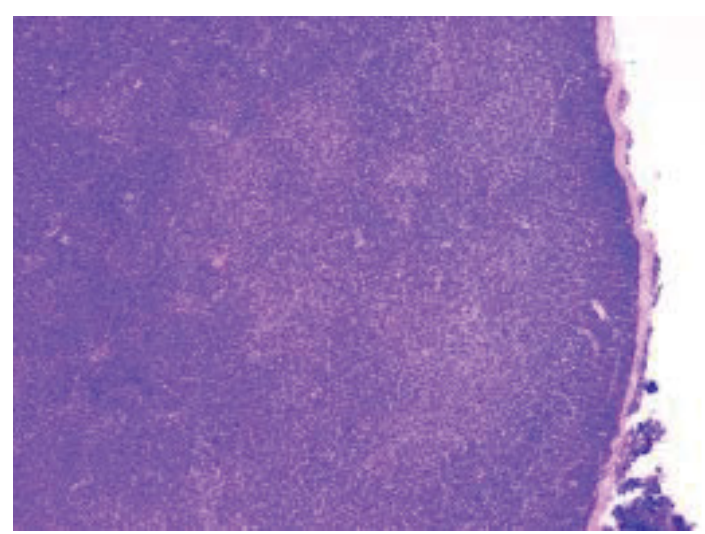

Figure 6: SLL-Obliterated nodal architecture with monotonous lymphocytes [25].

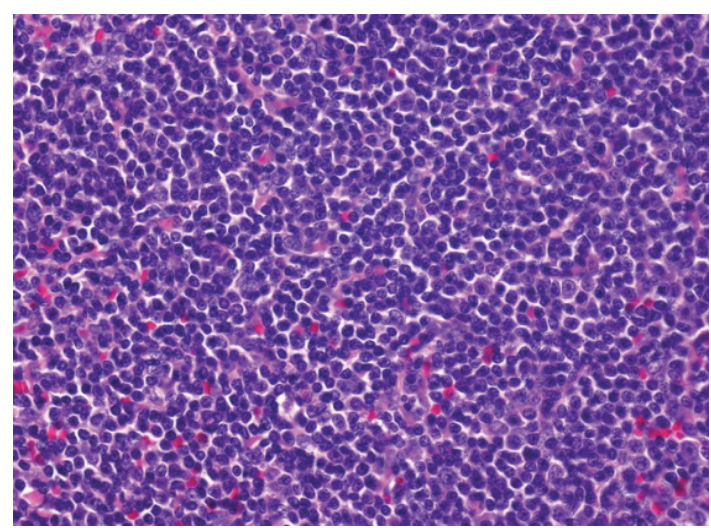

Figure 7: SLL-massive percolation of mature lymphocytes [21].

be of the $\mathrm{B}$ cell type. Monoclonal immunoglobulins such as the immunoglobulin $\mathrm{M}$ and $\mathrm{D}$ (IgM and $\operatorname{IgD}$ ) may be localized on the cell surface. The malignant B lymphocytes may differ from the cellular component of the follicular lymphoma which may demonstrate a pertinent, brighter intense staining reaction with appropriate aggregation of antibodies, along with a diminished concentration of the complement receptors [2]. An immune reactivity for CD20+, CD5+, CD23+, CD43+ and CyclinD1 may be exemplified. A genetic

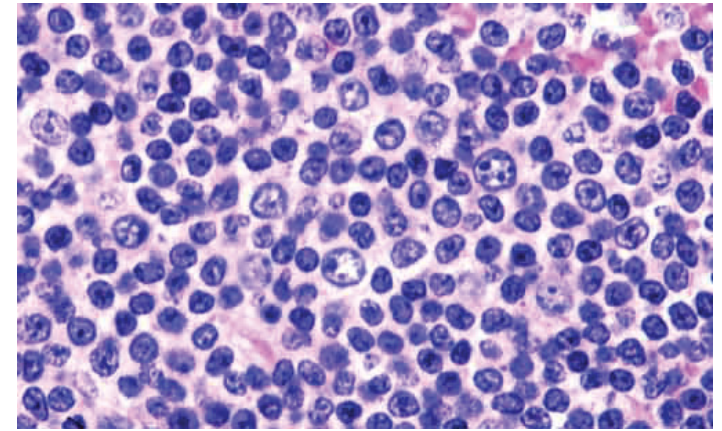

Figure 8: SLL-proliferation centre depicting large lymphoid cells with conspicuous nucleoli [26].

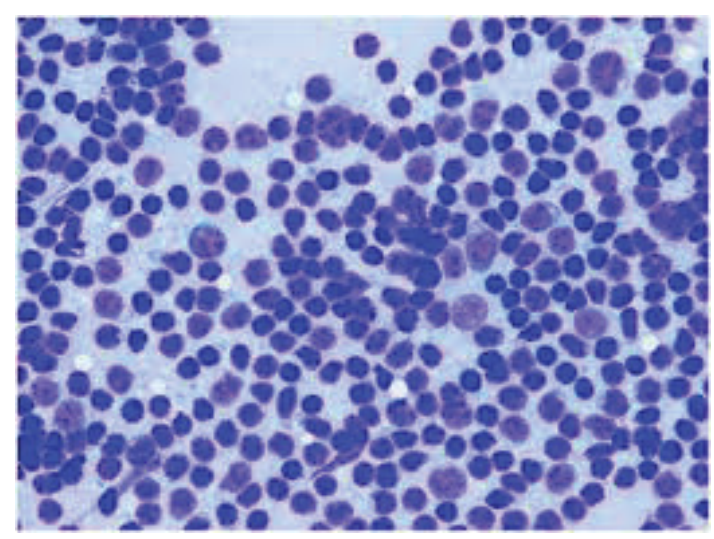

Figure 9: SLL-dense dispersion of mature lymphocytes aspiration cytology [27].

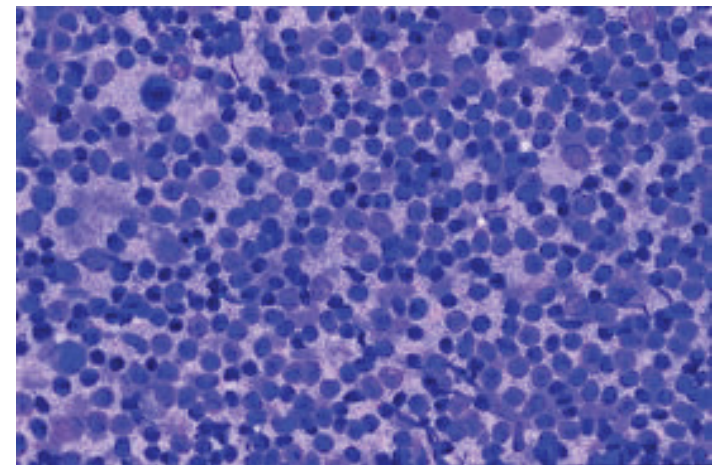

Figure 10: SLL-intensely stained and uniformly disseminated lymphocytes [28].

rearrangement of immunoglobulin G (IgG) heavy and light chains may be detected by molecular methodology [11]. Trisomy 12 may be discerned in one third of the instances, which indicates a poor prognosis. Aberrations of $13 \mathrm{q}$ associated with a favourable outcome may be delineated in one fourth individuals [2]. Somatic hypermutation of the immunoglobulin gene accompanied by a non-reactive, substitutive Zap 70 may be employed to analyse instances with a superior therapeutic outcome [12]. SLL may enunciate Reed Sternberg like cells, thus simulating a Hodgkin's Lymphoma, as a consequence of an Epstein Barr 


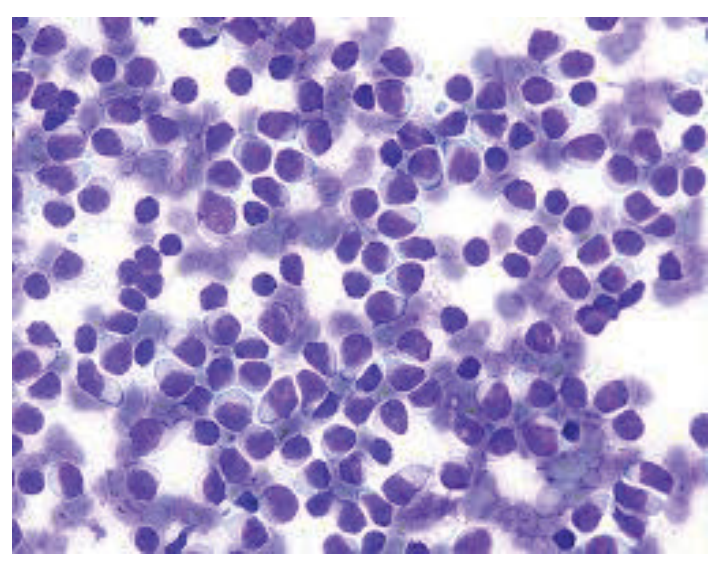

Figure 11: SLL-collection of small, mature lymphocytes-aspiration cytology [29].

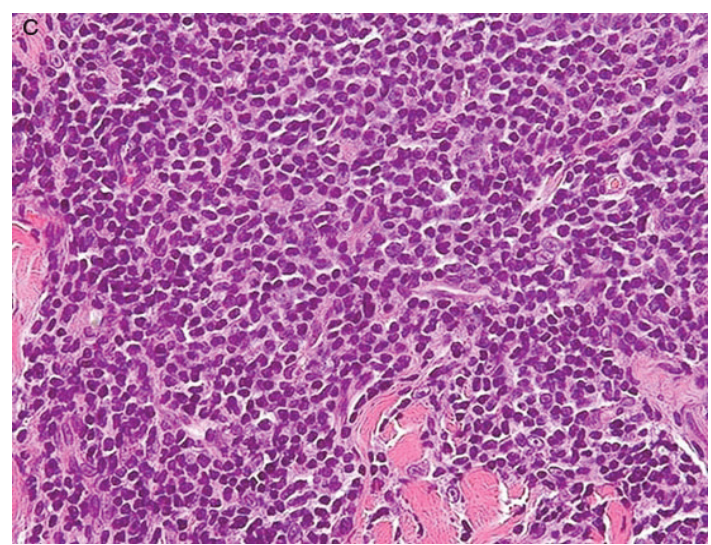

Figure 12: SLL-aggregates of small lymphocytes with accumulated paraprotein [30].

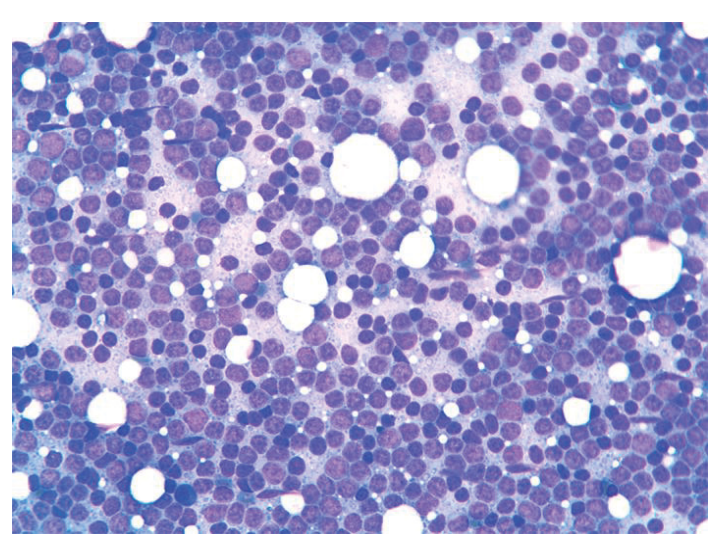

Figure 13: SLL- compact, abundant, mature small lymphocytesaspiration cytology [31].

viral intervention [2]. A blastic, histiocytic or a malignant conversion with enlarged cells may manifest with SLL or CLL. When occurring in a backdrop of a CLL, the phenomenon may be designated as "Richter's syndrome" and signifies an accelerated clinical course with a decline

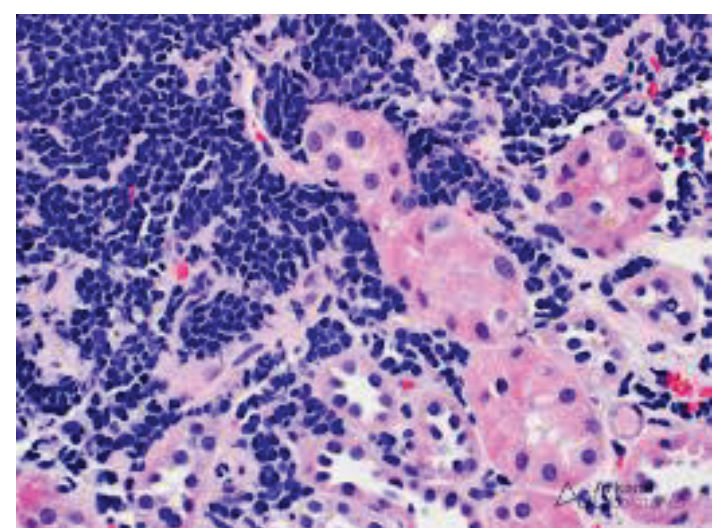

Figure 14: SLL cohesive, monotonous lymphocytes interspersed with renal glomeruli [32].

in the outcome [2,3]. Constitutional symptoms such as fever, lymph node enlargement, weight loss, abdominal pain with hepatomegaly and/or splenomegaly may be discerned $[3,4,13]$. The lymph nodes and the bone marrow may be the preliminary organs to be implicated. The enlarged, transformed cells may display identical immunoglobulin light and heavy chain restrictions as the SLL. The appearance of a "Richter's Syndrome" may be considered a "dedifferentiation" of the pre-existing neoplasm, rather than an emergence of a secondary malignancy $[2,5,14]$

\section{Prognostic Elements}

Molecular attributes which may elucidate unfavorable outcomes in CLL incorporate:

- FISH analysis of various cytogenetic aberrations such as chromosomal deletion of $17 \mathrm{p}$ or 11q [7]

- Complicated cytogenetic anomalies

- An un-mutated germline configuration of Immunoglobulin Heavy Chain Variable (IGHV) region beneath 2\%. Somatic hypermutation of the IGHV gene may be a critical prognostic/ predictive indicator

- An immune reactive ZAP $70+$ above $20 \%$, an immune-reactive CD38+ above $30 \%$ or an immune-reactive CD49+

- Elevated serum $\beta 2$ microglobulin levels

- A TP53 mutation detectable on genetic sequencing. Nextgeneration sequencing (whole exome sequencing) may also recognize numerous recurrent mutations such as NOTCH1, SF3B1, DDX3X, and ATM

\section{Indications For Therapy}

Irrespective of the genetic modifications detected within the SLL, therapy may be commenced with the appearance of constitutional symptoms of leukemia. Categories within the international working group for treating preliminary and relapsing CLL (2008) may incorporate [6]:

- Massive or progressing lymph node enlargement greater than 10-centimeter magnitude along with intrinsic symptoms

- A mammoth spleen extending 6 centimeters beyond the coastal margin accompanied by constitutional symptoms 
- Gradual or progressive malfunction of the bone marrow [7]

- A decline in the whole blood counts (cytopenias) secondary to the leukemia (hemoglobin $<11 \mathrm{gm} / \mathrm{dl}$, platelets $<100000$ cells/ $\mu \mathrm{L})$. Symptoms arising specifically on account of leukemia such as fatigue or B symptoms including fever, loss of greater than $10 \%$ of the body weight in six months or night sweats [15]

- The emergence of inappropriately managed autoimmune hemolytic anemia or an idiopathic thrombocytopenic purpura, conditions that are inadequately alleviated with corticosteroids or adjunctive therapies

- Lymphocytic perpetuation with a progressive lymphocyte counts above $50 \%$ of the baseline/initial quantification within a twomonth duration or a Lymphocyte Doubling Time (LDT) below six months [16]

\section{Therapeutic Protocols}

- The chemotherapeutic agents employed may be fludarabine, bendamustine, pentostatin, cyclophosphamide, and chlorambucil

- The targeted monoclonal antibodies applicable for the lymphoma/ leukemia may be rituximab, ofatumumab, obinutuzumab, alemtuzumab, etc. $[1,17]$

- The targeted therapies indicated for the disorder may be ibrutinib, ABT 199, idelalisib, etc. Despite a high risk, individuals may not mandate a preliminary commencement of therapy

- An initial chemo-immunotherapy may be preferable in the young individuals

- The Objective Response Rate (ORR) and Progression Free Survival (PFS) may be superior with the administration of Fludarabine, Cyclophosphamide, and Rituximab (FCR), in contrast to combined Bendamustine and Rituximab (BR). The Overall Survival (OS) may not be adequately affected, although the survival ratios are under evaluation. Severe neutropenia or pancytopenia (84\%) and persistent infections (39\%) may be frequently elucidated with the employment of fludarabine, cyclophosphamide, and rituximab, especially in individuals beyond 65 years of age, which may also lack an elevated progression-free survival ratio. Bendamustine and rituximab may similarly demonstrate neutropenia/ pancytopenia (59\%) and perpetual infections (25\%)

\section{Accepted Therapeutic Combinations}

The preferred treatment for the young healthy patients with the absence of co-morbidities, a Cumulative Illness Rating Scale (CIRS) of $\leq 6$ and a creatinine clearance $\geq 70 \mathrm{ml} / \mathrm{min}$ or a lack of del17p may be a combination of chemotherapeutic drugs with monoclonal antibodies [15-18]. The various, commingled modalities incorporate.

- Fludarabine in conjunction with Cyclophosphamide and Rituximab (FCR)

- Bendamustine with Rituximab (BR)

- Fludarabine with Rituximab (FR)

- Pentostatin with Cyclophosphamide and Rituximab (PCR)

- Obinutuzumab may also be affiliated with chlorambucil. The treatment endorsed for the elderly, debilitated patients with a CIRS of $\geq 6$ and a creatinine clearance $\leq 70 \mathrm{ml} / \mathrm{min}$ with a lack of del $17 \mathrm{p}$ may be indicated by [1]

- The incorporation of the patient within clinical trials

- Chemotherapy administered along with monoclonal antibodies such as Bendamustine with Rituximab (BR) or obinutuzumab along with chlorambucil. Rituximab may also be employed with chlorambucil or fludarabine. Rituximab as a solitary agent may be efficacious as are cladribine and chlorambucil

- An orally bio-available, tiny molecule termed as the Ventoclax with an affinity for BCL2 may be employed for mono-therapy as a singular therapeutic agent [18]

- A specific and powerful BTK inhibitor such as the acalabrutinib with minimal effects on the genetic elucidation of TEC, EGFR and ITK signaling may be beneficial [19-21]

\section{Contemporary Therapeutic Standards For CLL}

The high-risk individuals lacking therapeutic initiation may mandate an observation. Ibrutinib may be the preferred therapy in patients depicting a chromosomal $17 \mathrm{p}$ deletion. The administration of chlorambucil in combination with a CD20 monoclonal antibody such as rituximab may be beneficial in elderly individuals. The young and healthy individuals, eligible for chemo-immunotherapy, may benefit from concomitant Fludarabine, Cyclophosphamide, and Rituximab (FCR) or Bendamustine and Rituximab (BR) [7]. Active disease burden with chromosomal del $17 \mathrm{p}$ may benefit from salvage therapy such as a Bruton tyrosine kinase inhibitor (ibrutinib) or a phosphoinositide 3 kinase inhibitor (idelalisib) in combination with rituximab. Relapsed/ Refractory disease with a chromosomal $17 \mathrm{p}$ deletion may be managed with ventoclax. The appearance of a Richter transformation; may necessitate an intensive chemo-immunotherapy followed by an allogeneic stem cell transplantation [7].

\section{Immune Therapy}

Immune deregulation occurring within leukemia may enhance the checkpoint receptors manifested by the $\mathrm{T}$ cells and appropriate ligands of the leukemia cells. Prohibition of the checkpoint may regulate the immune system as a consequence of an anti-leukemic outcome $[1,3]$. The Graft Versus Leukaemia (GVL) response may be potently elucidated with the CLL. Allogeneic hematopoietic cell transplantation may be beneficial. Chimeric Antigen Receptor (CAR) T cell therapy may be advantageous. The CD $3 \mathrm{~T}$ cells may be accrued and then mobilized through procedures such as binding, fusion, integration, transcription with protein expression and ultimately impregnated through the CAR cell membrane with a reactive or weak lymphoid cell depletion, followed by an adoptive $\mathrm{T}$ cell transfer to the patient $[1,3,4]$. The patient may then be investigated for the therapeutic outcome and disease response with a CT scan, a bone marrow biopsy with relevant immune histochemistry and a peripheral blood examination. A flow cytometry with the continuation of the CAR T cell therapy may be indicated. The peripheral blood and bone marrow aspirate may also be subjected to a Reverse Transcriptase-Polymerase Chain Reaction (RT PCR) and a flow cytometric assay $[1,4,19]$.

\section{Novel Therapies}

Contemporary agents for treating CLL incorporate [7]:

- Ibrutinib prohibits the bruton tyrosine kinase and may be administered as an oral agent, efficacious for the chromosome 
$17 \mathrm{p}$ deletion. It may display side effects such as atrial fibrillation, hemorrhage, multiple infections

- Ventoclax as an oral agent may implicate the BCL2 gene. Complications such as tumor lysis syndrome, multiple drug interactions, and bi/pancytopenias may arise.

The median 5-year survival of CLL may extend from 95\% to $18 \%$ across diverse risk groups and the therapeutic modalities approbated (Tables 1-7).

\begin{tabular}{|l|c|c|}
\hline & Favourable Outcome & Unfavourable Outcome \\
\hline DNA Sequencing (IGHV) & $2 \%$ mutation & $<2 \%$ mutation \\
\hline Flow Cytometry & \multicolumn{2}{|c|}{} \\
\hline CD38 & $<30 \%$ & $\geq 30 \%$ \\
\hline Zap 70 & $<20 \%$ & $\geq 20 \%$ \\
\hline
\end{tabular}

Table 1: Immunoglobulin Heavy Chain (IGHV) variable gene mutation [1].

\begin{tabular}{|l|l|l|}
\hline \multicolumn{1}{|c|}{ Unfavourable } & \multicolumn{1}{|c|}{ Neutral } & \multicolumn{1}{c|}{ Favourable } \\
\hline del $11 q$ & Normal & del 13q (as a sole abnormality) \\
\hline del17p & 12 & \\
\hline
\end{tabular}

Table 2: Interphase cytogenetics (FISH) [1].

\begin{tabular}{|l|l|l|}
\hline $\begin{array}{c}\text { Genetic } \\
\text { Mutation }\end{array}$ & Incidence & \multicolumn{1}{c|}{ Prognostic evaluation } \\
\hline $\begin{array}{l}\text { Trisomy } 12 \\
\text { or } 12+\end{array}$ & $10 \%-20 \%$ & Indeterminate impact \\
\hline Del(11q23) & $5 \%-20 \%$ & $\begin{array}{l}\text { Poor prognosis. Responsive to chemotherapy and } \\
\text { immunotherapy }\end{array}$ \\
\hline Del (13q14) & $51 \%-62 \%$ & Favourable outcomes with the solitary aberration \\
\hline Del (17p) & $\begin{array}{l}3 \% \text { to } 7 \% \\
\text { of untreated } \\
\text { patients }\end{array}$ & Poor prognosis. Resistant to chemotherapy (FC/FCR) \\
\hline
\end{tabular}

Table 3: Chromosomal anomalies elucidated in CLL/SLL $[1,3,5]$.

\begin{tabular}{|l|l|l|}
\hline Stage & \multicolumn{1}{|c|}{ Description } & $\begin{array}{c}\text { Probability } \\
\text { Ratio }\end{array}$ \\
\hline 0 & $\begin{array}{l}\text { Lymphocytosis, peripheral blood lymphocytes }>15000 \\
\text { cells } / \mu \mathrm{L} \text { and }>40 \% \text { lymphocytes in the bone marrow }\end{array}$ & Low \\
\hline I & Stage 0 with enlarged nodes & Intermediate \\
\hline II & Stage 0-I with splenomegaly, hepatomegaly or both & Intermediate \\
\hline III & Stage 0-II with haemoglobin $<11 \mathrm{gm} / \mathrm{dl}$ & High \\
\hline IV & Stage 0-III with platelets $<100,000 \mu \mathrm{L}$ & High \\
\hline
\end{tabular}

Table 4: Staging system for CLL/SLL (Rai) [3]

\begin{tabular}{|l|l|}
\hline \multicolumn{1}{|c|}{ Stage } & \multicolumn{1}{|c|}{ Description } \\
\hline A & $\begin{array}{l}\text { Haemoglobin }>10 \mathrm{gm} / \mathrm{dl}, \text { Platelets }>100,000 \mathrm{~mm}^{3} \text { and }<3 \\
\text { enlarged areas }\end{array}$ \\
\hline B & $\begin{array}{l}\text { Haemoglobin }>10 \mathrm{gm} / \mathrm{dl} \quad, \text { Platelets }>100,000 \mathrm{~mm}^{3} \text { and } \geq 3 \\
\text { enlarged areas }\end{array}$ \\
\hline C & $\begin{array}{l}\text { Haemoglobin }<10 \mathrm{gm} / \mathrm{dl}, \text { Platelets }<100,000 \mathrm{~mm}^{3} \text { and any number } \\
\text { of enlarged areas }\end{array}$ \\
\hline
\end{tabular}

Table 5: Staging system for CLL/SLL (Binet) [3].

\begin{tabular}{|l|l|l|l|}
\hline Variable & Adverse Factor & Grading & Risk Group \\
\hline Chromosome 17p & Deletion & 4 & Very high risk 7-10 \\
\hline TP53 & Mutated & 4 & \\
\hline IGHV status & Un-mutated & 2 & High risk 4-6 \\
\hline Serum $\beta 2$ microglobulin & $3.5 \mathrm{mg} / \mathrm{L}$ & 2 & Intermediate risk 2-3 \\
\hline Clinical Stage & Binet B/C or Rai 3/4 & 1 & \\
\hline Age in years & $>65$ & 1 & Low risk 0-1 \\
\hline Prognostic Score & & $0-10$ & \\
\hline
\end{tabular}

Table 6: CLL-International Prognostic Index (CLL-IPI) [7].

\begin{tabular}{|c|c|c|}
\hline $\begin{array}{c}\text { Stage } \\
\text { Limited }\end{array}$ & Involvement & Extra-nodal (E) status \\
\hline Stage I & $\begin{array}{l}\text { One node or a group of } \\
\text { adjacent nodes }\end{array}$ & $\begin{array}{l}\text { Single extra-nodal lesion without } \\
\text { nodal involvement }\end{array}$ \\
\hline Stage II & $\begin{array}{l}\text { Two or more lymph node } \\
\text { groups on the same side of the } \\
\text { diaphragm }\end{array}$ & $\begin{array}{l}\text { Stage I or II lymph node region } \\
\text { with limited contiguous nodal } \\
\text { involvement }\end{array}$ \\
\hline $\begin{array}{l}\text { Stage II } \\
\text { bulky }\end{array}$ & II as above with bulky mass & Not applicable \\
\hline \multicolumn{3}{|l|}{ Advanced } \\
\hline \multirow[t]{2}{*}{ Stage III } & $\begin{array}{l}\text { Nodes on both sides of } \\
\text { diaphragm. }\end{array}$ & Not applicable \\
\hline & $\begin{array}{l}\text { Nodes above the diaphragm } \\
\text { with spleen involvement }\end{array}$ & \\
\hline Stage IV & $\begin{array}{l}\text { Additional non-contiguous } \\
\text { extra-lymphatic involvement }\end{array}$ & Not applicable \\
\hline
\end{tabular}

Table 7: Modified Ann Arbor staging of Non-Hodgkin's lymphoma (Lugano) [4].

\section{Therapeutic Objectives}

The goal of neoadjuvant therapy is to augment the perception of tumor biology and molecular technology with an objective to enhance the therapeutic outcomes. Targeted treatment modules may be evolved to judiciously eradicate malignant clones of cells. Self-immunity may be enlisted to eradicate the disease. Successful results of the treatment options availed may be enhanced in combination with the neoadjuvant therapy. The therapeutic amalgamation may extend the remission with ultimate alleviation $[1,3,4]$.

\section{Conclusion}

CLL is a frequent adult B cell leukemia detected at a median age of 72 years. An ALC of mature-appearing lymphocytes greater than 5000 cells $/ \mu \mathrm{L}$ with a B lymphocyte immune reactivity categorizes the condition. SLL may be exemplified where the ALC remains below 5000 cells $/ \mu \mathrm{L}$. The genetic discrepancies detected on FISH enunciate a $17 \mathrm{p}$ deletion, $11 \mathrm{q}$ deletion, $12 \mathrm{q}$ trisomy, a normal genotype or a solitary chromosomal13 q deletion. An "inter-follicular SLL" may be denominated with preponderantly diffuse disease and circumscribed, benign lymphoid follicles restricted to the marginal zone, perifollicular or inter-follicular areas [2]. An estimated one-third of SLL may exemplify an extra-nodal invasion $[5,10]$. The categories of SLL with distinctive clinical attributes may be:

An increase in the lymphocyte count with absolute lymphocytosis (designated as CLL).

A definitive monoclonal gammopathy with an estimated 50\% instances enunciating lesions of the bone marrow.

A clinical scenario with an absent absolute lymphocytosis or monoclonal gammopathy, although hypogammaglobulinemia may coexist [2]. Therapeutic preferences may include Ibrutinib in patients with a chromosomal $17 \mathrm{p}$ deletion. Chlorambucil with a CD20 monoclonal antibody such as rituximab may benefit elderly patients. Young and healthy individuals may be administered concomitant Fludarabine, Cyclophosphamide, and Rituximab (FCR) or Bendamustine and Rituximab (BR). Active disease burden with deletion of chromosome $17 \mathrm{p}$ may benefit from salvage therapy with a bruton tyrosine kinase inhibitor (ibrutinb) or a phosphoinositide 3 kinase inhibitor (idelalisib) in combination with rituximab. Immune therapies such as an allogeneic hematopoietic cell transplantation or a Chimeric Antigen Receptor (CAR) T cell therapy may be advantageous in young patients with a lack of co-morbidities. Novel therapies such 
as ibrutinib or ventoclax may be administered as oral agents and may prove to be efficacious for the chromosome $17 \mathrm{p}$ deletion.

\section{References}

1. John M Pagel (2017) Chronic lymphocytic leukaemia/small lymphocytic lymphoma 2017 update Swedish Cancer Institute Seattle Washington.

2. Rosai and Ackerman's "Surgical Pathology" Tenth Edition.

3. NCCN (2017) Clinical practice guidelines in oncology Chronic Lymphocytic Leukaemia/Small Lymphocytic Lymphoma Version.

4. Lymphoma Research Foundation Understanding the CLL/SLL 2017.

5. Jahic A, Custendil SH (2017) Chronic lymphocytic leukemia (cll)/small lymphocytic lymphoma (SLL)-all the aspects. Arch Med Res 5.

6. Swerdlow SH, Campo E, Pileri SA, Harris NL, Stein H, et al. (2016) The 2016 revision of world health organization classification of lymphoid neoplasm. Blood 127: 2375-2390.

7. Taimur Sher (2018) CLL What do I need to know as an internist in 2018 Mayo Clinic.

8. Rawstron AC (2011) Monoclonal B cell lymphocytosis 2009 haematology an education program. $430-439$

9. Kuppens R (2005) Mechanism of B-cell lymphoma pathogenesis. Nat Rev Cancer 54: 251-262.

10. Redaelli A (2004) The clinical and epidemiological burden of chronic lymphocytic leukaemia. Eur J Cancer Care 13: 279-287.

11. Montserrat E (2008) Chronic lymphocytic leukaemia: A short overview. Ann Oncol 19: 320-325

12. Mao Z, Quintanilla-Martinez L, Raffeld M, Richter M, Krugmann J, et al. (2007) IgVH mutational status and clonality analysis of Richter' $s$ transformationdiffuse large B cell lymphoma and Hodgkin's lymphoma in association with B cell chronic lymphocytic leukaemia (B-CLL) represent two different pathways of disease evolution. Am J Surg Path 31: 1605-1614.
13. Dicker F, Schnittger S, Haferlach T, Kern W, Schoch C, et al. (2006) Immunostimulatory oligonucleotide induced metaphase cytogenetics detect chromosomal aberrations in $80 \%$ CLL patients: A study of $132 \mathrm{CLL}$ cases with correlation to FISH, Ig VH status and CD 38 expression. Blood 108: 3152-3160.

14. Baliakas P, Iskas M, Gardiner A, Davis Z, Plevova K, et al. (2014) Chromosomal translocation and karyotype complexity in chronic lymphocytic leukaemia: A systematic reappraisal of classic cytogenetic data. Am J Haematol 89: 249-255.

15. Jennifer Ann Woyach, Amy S Ruppert, Gerard Lozanski, Arletta Lozanski, Nyla A Heerema, et al. (2014) Association of disease progression on ibrutinib therapy with the acquisition of resistance mutations : A single centre experience of 267 patients. J Clin Oncol 32: 7010.

16. Philip A Thompson, William G Wierda, Alessandra Ferrajoli, Susan C Smith, Susan O'Brien, et al. (2014) Complex karyotype rather than del (17p) is associated with inferior outcomes in relapsed or refractory CLL patients treated with ibrutinib based regimens. Blood 124: 22.

17. Stilgenbauer S, Schnaiter A, Paschka P, Zenz T, Rossi M, et al. (2014) Gene mutations and treatment outcome inchronic lymphocytic leukaemia: Results from the CLL8 trial. Blood 123: 3247-3254.

18. Puente XS, Pinyol M, Quesada V, Conde L, Ordóñez GR. et al. ( 2011) Whole genome sequencing identifies recurrent mutations in chronic lymphocytic leukaemia. Nature 475: 101-105.

19. Gabor Kovacs, Sebastian Boettcher, Jasmin Bahlo, Sandra Kluth, Matthias Ritgen, et al. (2014) Value of minimal residual disease negative status at response evaluation in chronic lymphocytic leukaemia combined analysis of two phase III studies of the German CLL study group (GCLLSG). Blood 124: 23.

20. Strati $P$, Shanafelt TD (2015) Monoclonal B cell lymphocytosis and early stage chronic lymphocytic leukaemia, diagnosis, natural history and risk stratification. Blood 126: 454-462.

21. Hallek M, Cheson BD, Catovsky D, Caligaris Cappio F, Dighiero G, et al. (2008) Guidelines for the diagnosis and treatment of chronic lymphocytic leukaemia: A repost from the international workshop on Chronic Lymphocytic Leukaemia updating the National Cancer Institute Working Group 1996 guidelines. Blood 111: $5446-5456$ 